\title{
Cysteine-rich protein 61 suppresses cell invasion via down- regulation of matrix metalloproteinase-7 expression in the human gastric carcinoma cell line MKN-45
}

\author{
MITSUHIKO OSAKI $^{1,2}$, AIKO INABA ${ }^{3}$, KEI NISHIKAWA ${ }^{1}$, YUI SUGIMOTO ${ }^{1}$, \\ KOHEI SHOMORI ${ }^{3}$, TOSHIAKI INOUE ${ }^{4}$, MITSUO OSHIMURA ${ }^{1,2}$ and HISAO ITO ${ }^{3}$
}

\author{
${ }^{1}$ Division of Molecular Genetics and Biofunction, Department of Biomedical Science, Tottori University \\ Graduate School of Medical Science; ${ }^{2}$ Chromosome Engineering Research Center; Divisions of ${ }^{3}$ Organ Pathology, \\ and ${ }^{4}$ Human Genome Science, Faculty of Medicine, Tottori University, Tottori 683-8503, Japan
}

Received March 4, 2010; Accepted May 20, 2010

DOI: $10.3892 / \mathrm{mmr} 00000322$

\begin{abstract}
Cysteine-rich protein 61 (CYR61) is a member of the CCN (CYR61/CTGF/NOV) family, which is associated with progression in a variety of human cancers. Our previous study confirmed that the expression levels of CYR61 protein were decreased in gastric carcinoma compared to non-tumoral mucosa as determined by proteome analysis. Histological research also showed that the reduction in CYR61 expression was significantly correlated with cellular invasiveness and inversely correlated with matrix metalloproteinase-7 (MMP-7/ matrilysin) expression in human gastric carcinoma. We examined the cause of CYR61 down-regulation in a human gastric carcinoma cell line, MKN-45. Lower expression of CYR61, but no genetic or epigenetic alterations of the gene, were observed. We then examined the correlation between CYR61 protein and MMP-7 expression and cellular invasiveness in MKN-45 cells. CYR61 was secreted from CYR61 expression-vector-transfected 293T cells, and the supernatant was added to MKN-45 cells. The expression level of MMP-7 was reduced by treatment of the supernatant, including CYR61, in a dose-dependent manner. An invasion assay showed that the cellular invasiveness of MKN-45 was significantly suppressed by the transfection of CYR61 expression vector compared to transfection with a control vector. Taken together, these results raise the possibility that CYR61 suppresses cell invasion at least partly via the downregulation of MMP-7 expression in human gastric carcinoma cells.
\end{abstract}

Correspondence to: Dr Mitsuhiko Osaki, Division of Molecular Genetics and Biofunction, Department of Biomedical Science, Tottori University Graduate School of Medical Science, Tottori University, 86 Nishi-cho, Yonago, Tottori 683-8503, Japan

E-mail: osamitsu@med.tottori-u.ac.jp

Key words: cysteine-rich protein 61, gastric carcinoma, cell invasion, matrix metalloproteinase-7

\section{Introduction}

Gastric carcinoma remains one of the major causes of cancerrelated death worldwide (1), particularly in Japan (2). Our previous study identified cysteine-rich protein 61 (CYR61) as a protein that is down-regulated in cancer lesions compared to non-tumoral mucosa, as determined by proteome analysis using resected human stomach tissue specimens (3). CYR61, a member of the CCN (CYR61/CTGF/NOV) family, was identified as a growth-factor-inducible immediate-early gene (4). The CCN family includes CCN1/CYR61, CCN2/ CTGF (connective tissue growth factor), CCN3/NOV (nephroblastoma-overexpressed), CCN4/WISP-1 (Wnt-1induced secreted proteins), CCN5/WISP-2 and CCN6/ WISP-3 (5). These are secreted proteins that associate with the extracellular matrix (ECM) and act as ligands of distinct integrins along with the other $\mathrm{CCN}$ members (5-7). It has been reported that CYR61 regulates normal cell adhesion, migration, proliferation and angiogenesis, and is involved in carcinogenesis (8-11). CYR61 expression was found to be up-regulated and to stimulate tumor progression in breast cancer, pancreatic cancer and gliomas (12-16). On the other hand, the expression of CYR61 was found to be downregulated in prostate cancer, endometrial cancer and non-small cell lung carcinoma, suggesting that CYR61 exerts variable effects in a variety of tumors (17-19).

Our previous study showed that a reduction in CYR61 expression was significantly correlated with clinicopathological features, such as histological differentiation, depth of invasion, lymphatic invasion, venous invasion, lymph node metastasis and clinical stage (20). The study also revealed that the expression of CYR61 is inversely correlated with that of matrix metalloproteinase-7 (MMP-7/matrilysin) (20). The matrix metalloproteinases (MMPs) comprise an endopeptidase family of over 25 members, which include collagenases, gelatinases, stromelysins and membrane-type MMP. MMP-2, -7 and -9 are mainly reported to be associated with gastric carcinoma (21-23). Among the MMPs, MMP-7 was identified as the only differentially overexpressed gene in gastric carcinoma cells in comparison to the normal cells as determined by DNA 
microarray analysis (24). Thus, our previous data suggested that CYR61 regulates the expression of MMP-7 and that the down-regulation of CYR61 may facilitate cancer cell invasion via MMP-7 activation in human gastric cancer.

In this study, we examined whether i) genetic or epigenetic alteration was associated with the down-regulation of CYR61, ii) the expression of MMP-7 was suppressed in the presence of CYR61 in a dose-dependent manner and iii) the forced expression of CYR61 inhibits cell invasion in a human gastric cancer cell line, MKN-45, in an in vitro study.

\section{Materials and methods}

CYR61 expression vector. A full-length CYR61 cDNA fragment was excised using EcoRI and NotI from pCMV-SPORT6/ CYR61 (Invitrogen, Carlsbad, CA, USA). This fragment was ligated into the same site as pcDNA3 mammalian expression vector (Invitrogen). After verifying the orientation of the insert, the constructed vector (pcDNA3-CYR61) was transfected into 293T or MKN-45 cells.

Cell culture. MKN-45 (a human gastric carcinoma cell line) and 293T (a human embryonic kidney cell line) were used in this study. Both cell lines were cultured in Dulbecco's modified Eagle's medium (DMEM; Nissui, Tokyo, Japan) containing $10 \%$ fetal bovine serum (FBS), $100 \mathrm{U} / \mathrm{ml}$ penicillin, $100 \mu \mathrm{g} / \mathrm{ml}$ streptomycin and $292 \mu \mathrm{g} / \mathrm{ml} \mathrm{L}(+)$-glutamine in a humidified $5 \% \mathrm{CO}_{2}$ incubator at $37^{\circ} \mathrm{C}$. MKN-45 cells were also cultured for $48 \mathrm{~h}$ in the presence of $1 \mu \mathrm{M} \mathrm{5'-aza-2'-deoxycytidine}$ (5'-aza), an inhibitor of DNA methyltransferase, or $500 \mathrm{nM}$ trichostatin A (TSA), an inhibitor of histone deacetylase.

Sequence analysis. Total RNA was extracted from each gastric carcinoma cell line using the RNeasy Mini kit (Qiagen, Hilden, Germany), in accordance with the manufacturer's instructions. RNA $(5 \mu \mathrm{g})$ was reverse-transcribed using a cDNA Synthesis kit (TaKaRa, Otsu, Japan) with $0.25 \mu \mathrm{g}$ of $\operatorname{pd}(\mathrm{T})^{12-18}$, in accordance with the manufacturer's instructions. The CYR61 cDNA sequence was analyzed using ABI PRISM. The primers and inner primers used in this analysis were as follows: 1F, 5'-CTTGTTGGCGTCTTCGTCG-3'; 1R, 5'-AGA CCTTACAGCAGCCGCAG-3'; 2F， 5'-TGCTGTAAGGTC TGCGCCAAGC-3'; 2R, 5'-CTTGGGGACACAGAGGAA TGCA-3'; 3F, 5'-TTCCTCTGTGTCCCCAAG-3'; 3R, 5'-CTGCCTTTTCCAACTGCA-3'; 4F, 5'-CAGTTGGAA AAGGCAGCTCACT-3'; 4R, 5'-CCGCACCTCACAAAT CCGGGTT-3'; 5F，5'-AACCCGGATTTGTGAGGTGCG-3'; 5R， 5'-AACCGCATCTTCACAGTCCT-3'; 6F， 5'-AGG ACTGTGAAGATGCGGTT-3'; 6R, 5'-GTTTGTCTAGGT GTGCCC-3'. The sequence data were compared to DDBJRELEASE: BC001271 (CYR61 cDNA).

Treatment of supernatant, including secreted CYR61 from $293 T$ cells in MKN-45 cells. 293T cells were seeded at $1 \times 10^{5}$ cells/well in 6-well plates. pcDNA3-CYR61 $(2 \mu \mathrm{g})$ and $6 \mu \mathrm{l}$ of FuGENE HD Transfection Reagent (Roche Applied Science, Basel, Switzerland) were mixed into $100 \mu \mathrm{l}$ of Opti-MEM I Reduced-Serum medium (Invitrogen). The transfection protocol was carried out in accordance with the manufacturer's instructions (Roche Applied Science). pcDNA3
$(2 \mu \mathrm{g})$ was used as a control. After $48 \mathrm{~h}$ of incubation with DMEM, the medium was changed to new serum-free DMEM and incubated for an additional $24 \mathrm{~h}$, then the supernatants were collected.

MKN-45 cells were plated at $5 \times 10^{5}$ cells/well in 6-well plates. After overnight incubation, the medium was removed and $1.2 \mathrm{ml}$ of 293T cell supernatants (including 0, 20, 40 and $80 \%$ supernatants from pcDNA3 transfectants or those from pcDNA3-CYR61 transfectants, in which secreted CYR61 was present) and $800 \mu \mathrm{l}$ of DMEM were added. Final FBS concentrations were conditioned to $10 \%$. Cells were incubated for an additional $72 \mathrm{~h}$.

Western blotting. Cells were washed with PBS(-) twice, harvested and solubilized in lysis buffer $(20 \mathrm{mM}$ Tris- $\mathrm{HCl}$, pH 7.4, $150 \mathrm{mM} \mathrm{NaCl}, 0.1 \%$ SDS, $1 \%$ sodium deoxycholate, $1 \%$ Triton X-100, $1 \mathrm{mM}$ PMSF, $5 \mu \mathrm{g} / \mathrm{ml}$ aprotinin and $1 \mu \mathrm{g} /$ $\mathrm{ml}$ leupeptin). Lysate was centrifuged at 14,500 rpm for $5 \mathrm{~min}$ at $4^{\circ} \mathrm{C}$. The protein concentration was determined using the Bio-Rad Protein assay (Bio-Rad Lab, Hercules, CA, USA) with bovine serum albumin (Wako, Osaka, Japan) as the standard. Protein $(50 \mu \mathrm{g})$ was separated by electrophoresis on $12 \%$ SDS-polyacrylamide gels and electrotransferred to a polyvinylidene difluoride filter (Millipore, Billerica, MA, USA). The membranes were blotted with anti-CYR61 polyclonal antibody (1:100; Santa Cruz Biotechnology, Santa Cruz, CA, USA) and anti-MMP-7 monoclonal antibody (1:50; Daiichi Fine Chemicals, Takaoka, Japan) or anti- $\beta$-actin monoclonal antibody (1:2,000; Sigma, St. Louis, MO, USA). Blots were developed with peroxidase-labeled anti-goat antibody (1:2,000; Santa Cruz Biotechnology) or peroxidase-labeled anti-mouse antibody (1:2,000; MBL, Nagoya, Japan) using an enhanced chemiluminescence system (ECL detection system; GE Healthcare, Buckinghamshire, UK).

Cell invasion assay. A cell invasion assay was performed using BD BioCoat Matrigel Invasion Chambers (BD Biosciences, CA, USA). MKN-45 cells were transfected with pcDNA3CYR61 or pcDNA3. After overnight incubation, cells were plated at $5 \times 10^{4}$ cells/well in 24-well chambers. The protocol was conducted according to the manufacturer's instructions (BD Biosciences). Invaded cells were mixed with calcein AM fluorescent dye (BD Biosciences) and incubated for $1 \mathrm{~h}$; then, the fluorescence of each well was measured at $535 \mathrm{~nm}$ using a plate reader.

\section{Results}

Genetic or epigenetic alterations of CYR61 in the human gastric carcinoma cell line MKN-45. To reveal the cause of the low expression of CYR61 protein in MKN-45 cells, CYR61 cDNA was examined by direct sequencing and a search for mutations was conducted within the CYR61 coding region. There were no mutations in the cell line compared to DDBJ-RELEASE: BC001271 (CYR61 cDNA) (data not shown). Next, alterations in epigenetic regulation were examined. Cells were treated with $1 \mu \mathrm{M} 5$ '-aza, an inhibitor of DNA methyltransferase, or $500 \mathrm{nM}$ TSA, an inhibitor of histone deacetylase, for $48 \mathrm{~h}$. However, Western blotting showed that there were no differences in CYR61 protein expression 


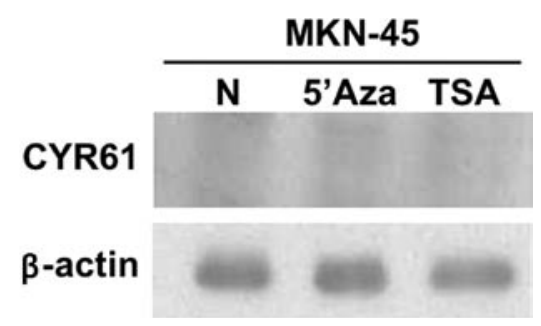

Figure 1. Western blot analysis of CYR61 in MKN-45 cells. Expression of CYR61 was not observed in MKN-45 cells. Treatment with an inhibitor of DNA methyltransferase or an inhibitor of histone deacetylase for $48 \mathrm{~h}$ did not affect the expression of CYR61 protein. $\mathrm{N}$, no treatment; 5'aza, $1 \mu \mathrm{M}$ 5'-aza-2'-deoxycytidine; TSA, $500 \mathrm{nM}$ trichostatin A.

A

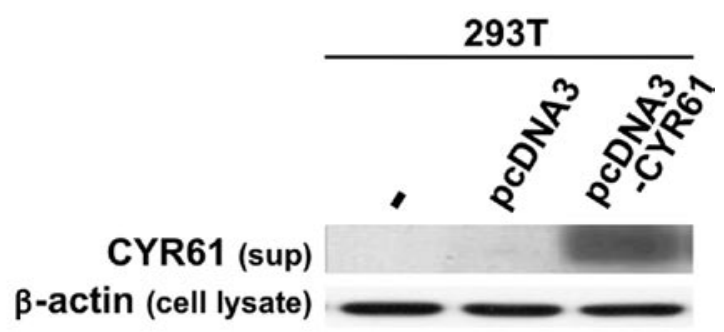

B

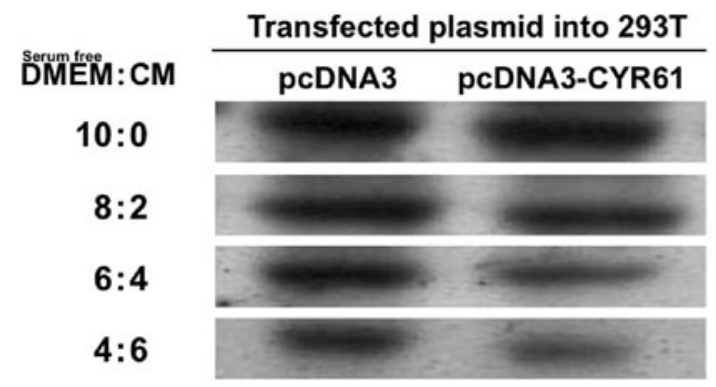

Figure 2. Treatment of secreted CYR61 protein suppressed MMP-7 expression in MKN-45 cells. (A) Western blot analysis showed CYR61 secretion into supernatant (sup) from 293T cells transfected with CYR61 expression vector (pcDNA3-CYR61), but no secretion from cells transfected with the control vector (pcDNA3). Each supernatant was used as a conditioned medium. The conditioned media were collected from either pcDNA3- or pcDNA3-CYR61transfected 293T cells after being grown in serum-free media for $24 \mathrm{~h}$. (B) Western blot analysis revealed that MMP-7 expression is suppressed by treatment of conditioned media, including secreted CYR61 protein in a dosedependent manner in MKN-45 cells. CM, conditioned media.

between the control, 5'-aza-treated and TSA-treated groups (Fig. 1). These results suggest that CYR61 expression was not epigenetically suppressed in MKN-45 cells.

CYR61 down-regulates MMP-7 expression in MKN-45 cells. CYR61 protein is known to be a secreted protein. Histological examination in our previous study suggested that CYR61 was secreted from endocrine cells in human gastric mucosa (20). Additionally, the reduction in CYR61 expression in gastric carcinoma was found to be correlated with more malignant phenotypes, including depth of invasion, lymphatic invasion, venous invasion, lymph node metastasis and clinical stage (20). These results strongly indicate that the down-regulation of CYR61 enhanced cellular invasion by gastric carcinoma cells. In human gastric carcinoma, MMP-7 is known as a major factor promoting cell invasion into the ECM (25-30). To
$\mathbf{A}$

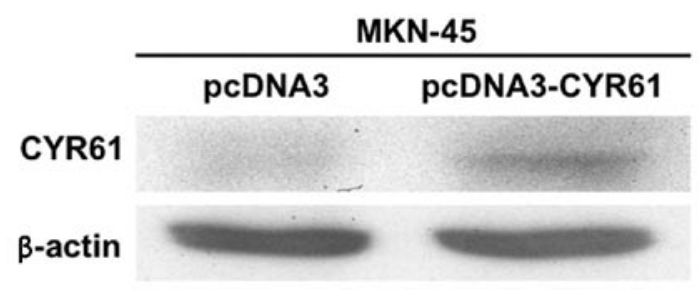

B

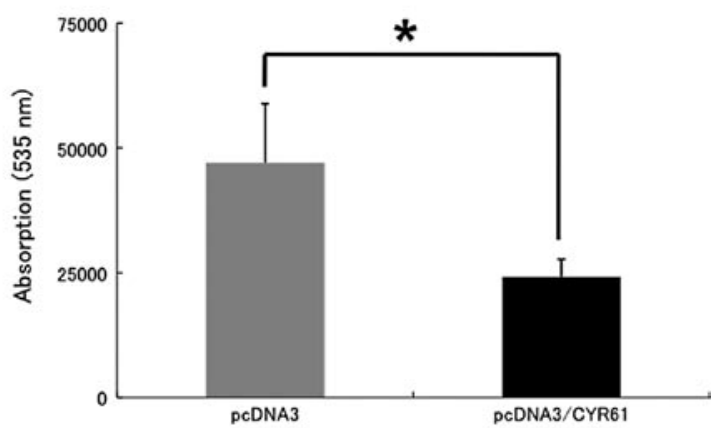

Figure 3. CYR61 inhibited the cell invasiveness of MKN-45 in vitro. (A) Exogenous CYR61 expression was induced by the transfection of pcDNA3CYR61 into MKN-45, but not pcDNA3. (B) Matrigel invasion assay was performed on MKN-45 transfected with either pcDNA3-CYR61 or pcDNA3. Invaded cells were treated with calcein-AM fluorescent dye and incubated for $1 \mathrm{~h}$; then, the fluorescence of each well was measured at $535 \mathrm{~nm}$ using a plate reader. Data are presented as the means of triplicate determinations; error bars represent the SD. *Statistically significant difference $(\mathrm{P}<0.05)$ between pcDNA3-CYR61- and pcDNA-transfected MKN-45 cells.

reveal whether treatment with CYR61 protein affects MMP-7 expression, MKN-45 cells were cultured in conditioned media, including CYR61 protein secreted from pcDNA3-CYR61transfected 293T cells (Fig. 2A). Western blotting showed that MMP-7 expression in MKN-45 cells treated with CYR61 protein was decreased in a dose-dependent manner (Fig. 2B), indicating that MMP-7 expression was down-regulated by secretory CYR61 protein in MKN-45 cells.

Finally, a cell invasion assay was performed to confirm the effect of CYR61 on cell invasion in MKN-45 cells. CYR61 protein was expressed in transient pcDNA3-CYR61-transfected MKN-45 cells, but not in pcDNA3-transfected cells (Fig. 3A). It was demonstrated that the number of invaded cells was significantly suppressed in pcDNA3-CYR61-transfected cells compared to pcDNA3-transfected cells in MKN-45 ( $\mathrm{P}<0.05$, Fig. 3B).

\section{Discussion}

The present study examined the cause of reduced CYR61 expression in a human gastric carcinoma cell, MKN-45, by examining genetic and epigenetic regulation. On the basis of direct sequencing, it was determined that there was no mutation in the coding region of the CYR61 gene in MKN-45 cells. We then examined whether epigenetic disorders explain the reduced CYR61 expression. To date, it has been reported that a number of tumor suppressor genes, such as RUNX3 $(31,32)$, DAP kinase (32), pl6 (33), hMLHI (34) and TSLCl (35), amongst others, are silenced by promoter hypermethylation in gastric carcinoma. Our findings suggested that an epigenetic disorder did not affect the down-regulation of CYR61 expres- 
sion in the MKN-45 cell line and that other mechanisms may be associated with the suppression of CYR61 expression in human gastric cancer.

In our previous study, the reduced expression of CYR61 in gastric carcinoma was significantly associated with several clinicopathological factors, including depth of invasion, vessel invasion, lymph node metastasis and clinical stage. Moreover, immunohistochemical analysis strongly suggested that the expression of CYR61 was inversely correlated with MMP-7 expression in the invasive front of gastric carcinoma (20). Western blotting in the present study also showed an inverse correlation between the expression of CYR61 and MMP-7 in human gastric carcinoma cell lines, supporting the previous immunohistochemical results. We therefore examined this correlation in vitro. Our data demonstrated that treatment with CYR61 down-regulates the expression of MMP-7 in a dosedependent manner in MKN-45. In addition, these in vitro data were consistent with the previous in vivo data obtained using clinical samples. However, how CYR61 regulates the expression of MMP-7 cells remains to be determined. MMP-7 has been considered a major factor in gastric carcinoma invasion (25-30) through the activation of MMP-2 and -9, and thus plays a central role in the degradation of the extracellular matrix, including type IV collagen (36). To date, WISP-1, a member of the CCN family, has been shown to downregulate MMP-1 through the inhibition of Rac activation in human lung cancer cells (37). This suggests that other CCN family proteins, including CYR61, may down-regulate MMP-7 expression. Mitra et al reported that the ligation of cell surface $\alpha_{5} \beta_{1}$ integrin by $\alpha_{5}$ monoclonal antibody leads to the expression and activation of MMP-2 and -7 in B16F10 melanoma cells (38). Furthermore, Yamamoto et al reported that $\mathrm{E} 1 \mathrm{AF}$, ets-related transcriptional factor, the expression of which induces the expression of MMP-7, plays a key role in the progression of gastric cancer (39). These data suggest a possible mechanism of MMP-7 down-regulation by treatment with CYR61 protein, which may exert its effect through $\alpha_{5} \beta_{1}$ integrin activity, thus inhibiting E1AF activation.

By contrast, Lin et al reported that CYR61 induces gastric carcinoma cell invasion via the activation of the integrin $\alpha_{\mathrm{v}} \beta_{3} /$ $\mathrm{NF}-\kappa \mathrm{B} /$ cyclooxygenase-2 signaling pathway (40), and that the elevated expression of CYR61 enhances peritoneal dissemination through integrin $\alpha_{2} \beta_{1}(41)$. Although these reports do not necessarily coincide with those of this study, the discrepancy may in part be explained by the differences in interacting integrins and the cell line used in each study. Further studies are required to elucidate in detail the mechanism by which CYR61 protein regulates MMP-7 expression in not only MKN-45, but also in other gastric cancer cell lines.

In summary, this study showed that CYR61 down-regulates MMP-7 expression and inhibits cancer cell invasion by MKN-45 cells. We conclude that CYR61 may be a critical protein for the inhibition of gastric cancer cell invasion.

\section{Acknowledgements}

This study was supported, in part, by a Grant-in-Aid for Scientific Research from the Ministry of Education, Culture, Sports, Science and Technology of Japan (grant no. 19790260).

\section{References}

1. Fuchs CS and Mayer RJ: Gastric carcinoma. N Engl J Med 333: 32-41, 1995.

2. Statistics and Information Department, Minister's Secretariat, Ministry of Health. Vital statistics 1998 Japan (in Japanese). Vol 1. Health and Statistics Association, Tokyo, pp284-290, 2000.

3. Nishigaki R, Osaki M, Hiratsuka M, et al: Proteomic identification of differentially-expressed genes in human gastric carcinomas. Proteomics 5: 3205-3213, 2005.

4. O'Brien TP, Yang GP, Sanders L and Lau LF: Expression of cyr61, a growth factor-inducible immediate-early gene. Mol Cell Biol 10: 3569-3577, 1990.

5. Brigstock DR: The connective tissue growth factor/cysteine-rich 61/nephroblastoma overexpressed (CCN) family. Endocr Rev 20: 189-206, 1999.

6. Lau LF and Lam SC: The CCN family of angiogenic regulators: the integrin connection. Exp Cell Res 248: 44-57, 1999.

7. Perbal B: NOV (nephroblastoma overexpressed) and the CCN family of genes: structural and functional issues. Mol Pathol 54: 57-79, 2001.

8. Babic AM, Kireeva ML, Kolesnikova TV and Lau LF: CYR61, a product of a growth factor-inducible immediate early gene, promotes angiogenesis and tumor growth. Proc Natl Acad Sci USA 95: 6355-6360, 1998.

9. Kireeva ML, Latinkic BV, Kolesnikova TV, Chen CC, Yang GP, Abler AS and Lau LF: Cyr61 and Fisp12 are both ECM-associated signaling molecules: activities, metabolism, and localization during development. Exp Cell Res 233: 63-77, 1997.

10. Kireeva ML, Mo FE, Yang GP and Lau LF: Cyr61, a product of a growth factor-inducible immediate-early gene, promotes cell proliferation, migration, and adhesion. Mol Cell Biol 16: 1326-1334, 1996

11. Grzeszkiewicz TM, Lindner V, Chen N, Lam SC and Lau LF: The angiogenic factor cysteine-rich 61 (CYR61, CCN1) supports vascular smooth muscle cell adhesion and stimulates chemotaxis through integrin alpha(6)beta(1) and cell surface heparan sulfate proteoglycans. Endocrinology 143: 1441-1450, 2002.

12. Tsai MS, Bogart DF, Castaneda JM, Li P and Lupu R: Cyr61 promotes breast tumorigenesis and cancer progression. Oncogene 21: 8178-8185, 2002.

13. Xie D, Yin D, Tong X, et al: Cyr61 is overexpressed in gliomas and involved in integrin-linked kinase-mediated Akt and betacatenin-TCF/Lef signaling pathways. Cancer Res 64: 1987-1996, 2004.

14. Menendez JA, Mehmi I, Griggs DW and Lupu R: The angiogenic factor CYR61 in breast cancer: molecular pathology and therapeutic perspectives. Endocr Relat Cancer 10: 141-152, 2003.

15. Tsai MS, Bogart DF, Li P, Mehmi I and Lupu R: Expression and regulation of Cyr61 in human breast cancer cell lines. Oncogene 21: 964-973, 2002.

16. Holloway SE, Beck AW, Girard L, Jaber MR, Barnett CC Jr, Brekken RA and Fleming JB: Increased expression of Cyr61 (CCN1) identified in peritoneal metastases from human pancreatic cancer. J Am Coll Surg 200: 371-377, 2005.

17. Pilarsky CP, Schmidt U, Eissrich C, et al: Expression of the extracellular matrix signaling molecule Cyr61 is downregulated in prostate cancer. Prostate 36: 85-91, 1998.

18. Chien W, Kumagai T, Miller CW, Desmond JC, Frank JM, Said JW and Koeffler HP: Cyr61 suppresses growth of human endometrial cancer cells. J Biol Chem 279: 53087-53096, 2004.

19. Tong X, Xie D, O'Kelly J, Miller CW, Muller-Tidow C and Koeffler HP: Cyr61, a member of CCN family, is a tumor suppressor in non-small cell lung cancer. J Biol Chem 276: 47709-47714, 2001.

20. Maeta N, Osaki M, Shomori K, Inaba A, Kidani K, Adachi H and Ito H: CYR61 down-regulation correlates with tumor progression by promoting MMP-7 expression in the human gastric carcinoma. Oncology 73: 118-126, 2007.

21. Honda M, Mori M, Ueo H, Sugimachi K and Akiyoshi T: Matrix metalloproteinase-7 expression in gastric carcinoma. Gut 39: 444-448, 1996.

22. Nomura H, Fujimoto N, Seiki M, Mai M and Okada Y: Enhanced production of matrix metalloproteinases and activation of matrix metalloproteinase 2 (gelatinase A) in human gastric carcinomas. Int J Cancer 69: 9-16, 1996.

23. Sier CF, Kubben FJ, Ganesh S, et al: Tissue levels of matrix metalloproteinases MMP-2 and MMP-9 are related to the overall survival of patients with gastric carcinoma. Br J Cancer 74: 413-417, 1996. 
24. Mori M, Mimori K, Yoshikawa Y, et al: Analysis of the geneexpression profile regarding the progression of human gastric carcinoma. Surgery 131: 39-47, 2002.

25. Yamashita K, Azumano I, Mai M and Okada Y: Expression and tissue localization of matrix metalloproteinase 7 (matrilysin) in human gastric carcinomas. Implications for vessel invasion and metastasis. Int J Cancer 79: 187-194, 1998.

26. Huachuan Z, Xiaohan L, Jinmin S, Qian C, Yan X and Yinchang Z: Expression of matrix metalloproteinase-7 involving in growth, invasion, metastasis and angiogenesis of gastric cancer. Chin Med Sci J 18: 80-86, 2003.

27. Zheng HC, Sun JM, Li XH, Yang XF, Zhang YC and Xin Y: Role of PTEN and MMP-7 expression in growth, invasion, metastasis and angiogenesis of gastric carcinoma. Pathol Int 53: 659-666, 2003.

28. Chen JQ, Zhan WH, He YL, Peng JS, Wang JP, Cai SR and Ma JP: Expression of heparanase gene, CD44v6, MMP-7 and nm23 protein and their relationship with the invasion and metastasis of gastric carcinomas. World J Gastroenterol 10: 776-782, 2004.

29. Kitoh T, Yanai H, Saitoh Y, et al: Increased expression of matrix metalloproteinase-7 in invasive early gastric cancer. J Gastroenterol 39: 434-440, 2004.

30. Aihara R, Mochiki E, Kamiyama Y, Ohno T, Asao T and Kuwano H: Matrilysin expression is a useful marker of submucosal invasion and lymph node metastasis in early stage signet ring cell carcinoma of the stomach. J Surg Oncol 93: 491-497, 2006.

31. Li QL, Ito K, Sakakura C, et al: Causal relationship between the loss of RUNX3 expression and gastric cancer. Cell 109: 113-124, 2002.

32. Waki T, Tamura G, Sato M, Terashima M, Nishizuka S and Motoyama T: Promoter methylation status of DAP-kinase and RUNX3 genes in neoplastic and non-neoplastic gastric epithelia. Cancer Sci 94: 360-364, 2003.
33. Hou P, Ji MJ, Shen JY, He NY and Lu ZH: Detection of p16 hypermethylation in gastric carcinomas using a seminested methylation-specific PCR. Biochem Genet 43: 1-9, 2005.

34. Endoh Y, Tamura G, Ajioka Y, Watanabe H and Motoyama T: Frequent hypermethylation of the hMLH1 gene promoter in differentiated-type tumors of the stomach with the gastric foveolar phenotype. Am J Pathol 157: 717-722, 2000.

35. Honda T, Tamura G, Waki T, et al: Hypermethylation of the TSLC1 gene promoter in primary gastric cancers and gastric cancer cell lines. Jpn J Cancer Res 93: 857-860, 2002.

36. Parsons SL, Watson SA, Brown PD, Collins HM and Steele RJ: Matrix metalloproteinases. Br J Surg 84: 160-166, 1997.

37. Soon LL, Yie TA, Shvarts A, Levine AJ, Su F and Tchou-Wong KM: Overexpression of WISP-1 down-regulated motility and invasion of lung cancer cells through inhibition of Rac activation. J Biol Chem 278: 11465-11470, 2003.

38. Mitra A, Chakrabarti J and Chatterjee A: Binding of alpha5 monoclonal antibody to cell surface alpha5betal integrin modulates MMP-2 and MMP-7 activity in B16F10 melanoma cells. J Environ Pathol Toxicol Oncol 22: 167-178, 2003.

39. Yamamoto H, Horiuchi S, Adachi Y, Taniguchi H, Nosho K, Min $\mathrm{Y}$ and Imai $\mathrm{K}$ : Expression of ets-related transcriptional factor E1AF is associated with tumor progression and overexpression of matrilysin in human gastric cancer. Carcinogenesis 25: 325-332, 2004.

40. Lin MT, Zuon CY, Chang CC, et al: Cyr61 induces gastric cancer cell motility/invasion via activation of the integrin/nuclear factor-kappaB/cyclooxygenase-2 signaling pathway. Clin Cancer Res 11: 5809-5820, 2005.

41. Lin MT, Chang CC, Lin BR, Yang HY, Chu CY, Wu MH and Kuo ML: Elevated expression of Cyr61 enhances peritoneal dissemination of gastric cancer cells through integrin alpha2beta1. J Biol Chem 282: 34594-34604, 2007. 